\title{
Primary intimal pulmonary artery sarcoma: A diagnostic challenge
}

\author{
Hans Scheffel, MD, ${ }^{a}$ Paul Stolzmann, MD, ${ }^{a}$ André Plass, MD, ${ }^{b}$ Achim Weber, MD, ${ }^{c}$ René Prêtre, MD, \\ Borut Marincek, MD, and Hatem Alkadhi, MD, ${ }^{a}$ Zurich, Switzerland
}

$\mathrm{P}$ rimary pulmonary artery sarcoma (PAS), which was first described by Mandelstamm in $1923,{ }^{1}$ is a rare entity that is often misdiagnosed as chronic pulmonary thromboembolism. ${ }^{2}$ The differentiation of these 2 diseases with similar clinical presentations is fundamental for the initiation of adequate therapy, which completely differs between the 2 conditions. ${ }^{3}$ PAS often has a fatal outcome because of insidious intravascular tumor growth with extension into the distal pulmonary artery system. Surgery and adjacent irradiation or chemotherapy are necessary to optimize the survival time that ranges between 14 and 18 months. ${ }^{4}$ Computed tomography (CT) can serve as one component in the diagnostic workup of PAS by providing characteristic imaging features that favor a PAS diagnosis over that of a thromboembolism. ${ }^{5}$ We present the CT findings of a patient with primary intimal PAS of the pulmonary trunk along with the intraoperative images and histopathologic findings.

\section{Clinical Summary}

A 38-year-old female patient with recurrent atypical chest pain was admitted to the University Hospital Zurich. Her medical history was remarkable with regard to a long-standing pulmonary arterial hypertension. She had undergone a commissurotomy of the pulmonic valve combined with infundibulectomy at the age of 4 years because of valvular and infundibular pulmonary stenosis. The physical examination revealed a systolic ejection murmur over the left border of the sternum. A 12-lead electrocardiography was consistent with a cor pulmonale. The laboratory data were normal. An external transthoracic echocardiography examination before admission showed a floating mass located at the bifurcation of the pulmonary trunk. A retrospective, contrast-enhanced, electrocardiographygated, dual-source CT scan of the chest was performed for further characterization of the lesion. A hypodense mass with mild contrast enhancement, measuring approximately $4 \times 4 \mathrm{~cm}$, that was attached to the pulmonary trunk bifurcation with extension into the proximal left pulmonary artery was demonstrated (Figure 1, $A$ ). An additional

From the Institute of Diagnostic Radiology, ${ }^{\text {a }}$ Clinic for Cardiovascular Surgery, ${ }^{\mathrm{b}}$ and Institute of Surgical Pathology, ${ }^{\mathrm{c}}$ University Hospital Zurich, Switzerland.

Received for publication Nov 9, 2007; accepted for publication Nov 12, 2007.

Address for reprints: Hatem Alkadhi, MD, Institute of Diagnostic Radiology, Department of Medical Radiology, Rämistrasse 100, 8091 Zurich, Switzerland (E-mail: hatem.alkadhi@usz.ch).

J Thorac Cardiovasc Surg 2008;135:949-50

0022-5223/ $\$ 34.00$

Copyright (C) 2008 by The American Association for Thoracic Surgery doi:10.1016/j.jtcvs.2007.11.041 mass with a similar appearance and pattern of contrast enhancement was found in the right pulmonary artery encompassing $2 \times 2 \mathrm{~cm}$. The masses had no sharp borders but were characterized by filiform tails extending into the pulmonary arteries presented as filling defects of the segmental and subsegmental segments (Figure 1, A). In addition, an infiltration of the mass through the wall of the pulmonary trunk with progression into the middle mediastinum was observed (Figure 1,B).

Subsequently, cardiac surgery was performed with resection of the 2 separate lesions. The first lesion started from the bifurcation and ended in the distal lobar arteries of the left lung (Figure 2, $A$ ). The second lesion started from the right pulmonary artery and extended into the superior lobar artery. A resection of the masses, similar to a thromboendarterectomy, was performed on both sides. Adjoining lymph nodes were also resected. The defects in the pulmonary artery wall were covered with a patch of xenopericardium. Histopathology revealed a poorly differentiated mesenchymal tumor with a heterogenous growth pattern including solid, epithelioid, and myxoid areas, as well as tumor necrosis and adherent thrombi (Figure 2, B). Postsurgery follow-up CT showed complete tumor resection of the central pulmonary artery system. The patient recovered from surgery and currently receives adjuvant chemotherapy.

\section{Discussion}

PAS and chronic pulmonary thromboembolic disease are challenging diseases that both appear as intraluminal filling defects in the pulmonary artery system on contrast-enhanced CT scans. However, $\mathrm{CT}$ is able to demonstrate some features that may help to differentiate these 2 diseases. CT indicators that favor the diagnosis of PAS over a chronic thrombus are occasional mild contrast enhancement of the mass and extravascular spread of the lesion. The penetration of the tumor into the mediastinum in our patient was particularly helpful in making the correct differential diagnosis of PAS.

Early diagnosis and differentiation of PAS from chronic pulmonary embolism are crucial for prolonging the survival of patients with PAS. The case presented demonstrates the possibilities of chest $\mathrm{CT}$ in the recognition of this rare clinical entity.

\section{References}

1. Mandelstamm M. Über primäre neubildungen des herzens. Virchows Arch. 1923;245:43-54.

2. Hoeper MM, Mayer E, Simonneau G, Rubin LJ. Chronic thromboembolic pulmonary hypertension. Circulation. 2006;113:2011-20.

3. Yi CA, Lee KS, Choe YH, Han D, Kwon OJ, Kim S. Computed tomography in pulmonary artery sarcoma: distinguishing features from pulmonary embolic disease. J Comput Assist Tomogr. 2004;28:34-9.

4. Manso L, Alvarez E, Quintela M, Cortes-Funes H, Hitt R. Primary pulmonary artery sarcoma: report of three cases and review of the literature. Clin Lung Cancer. 2007;8:277-81.

5. Cox JE, Chiles C, Aquino SL, Savage P, Oaks T. Pulmonary artery sarcomas: a review of clinical and radiologic features. J Comput Assist Tomogr. 1997;21:750-5. 

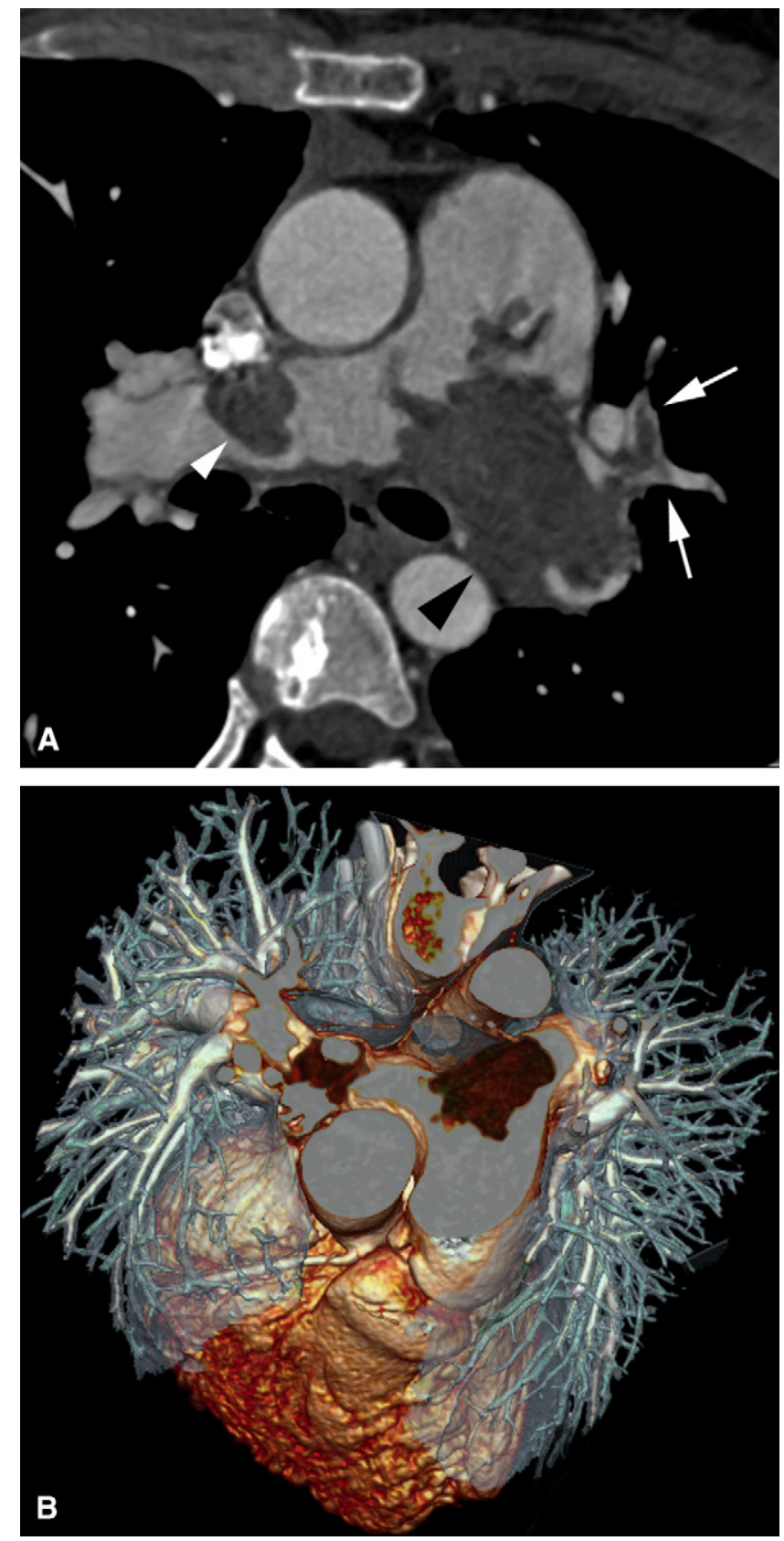

Figure 1. A, Contrast-enhanced transverse CT image shows the irregular mass occupying the bifurcation of the pulmonary trunk with extension into the left pulmonary artery and tumor spread in the lobar and segmental pulmonary arteries (white arrows). In addition, there is prospering of the tumor through the dorsal arterial wall with infiltration into the mediastinum (black arrowhead). Note the mass in the right pulmonary artery corresponding to a second tumor manifestation (white arrowhead). B, Threedimensional volume-rendered CT image of the patient's heart and adjacent lung with intersection at the level of the bifurcation of the pulmonary artery trunk demonstrates more clearly the $\mathbf{2}$ tumor parts (red) in the pulmonary trunk and arteries in relation to the surrounding structures.
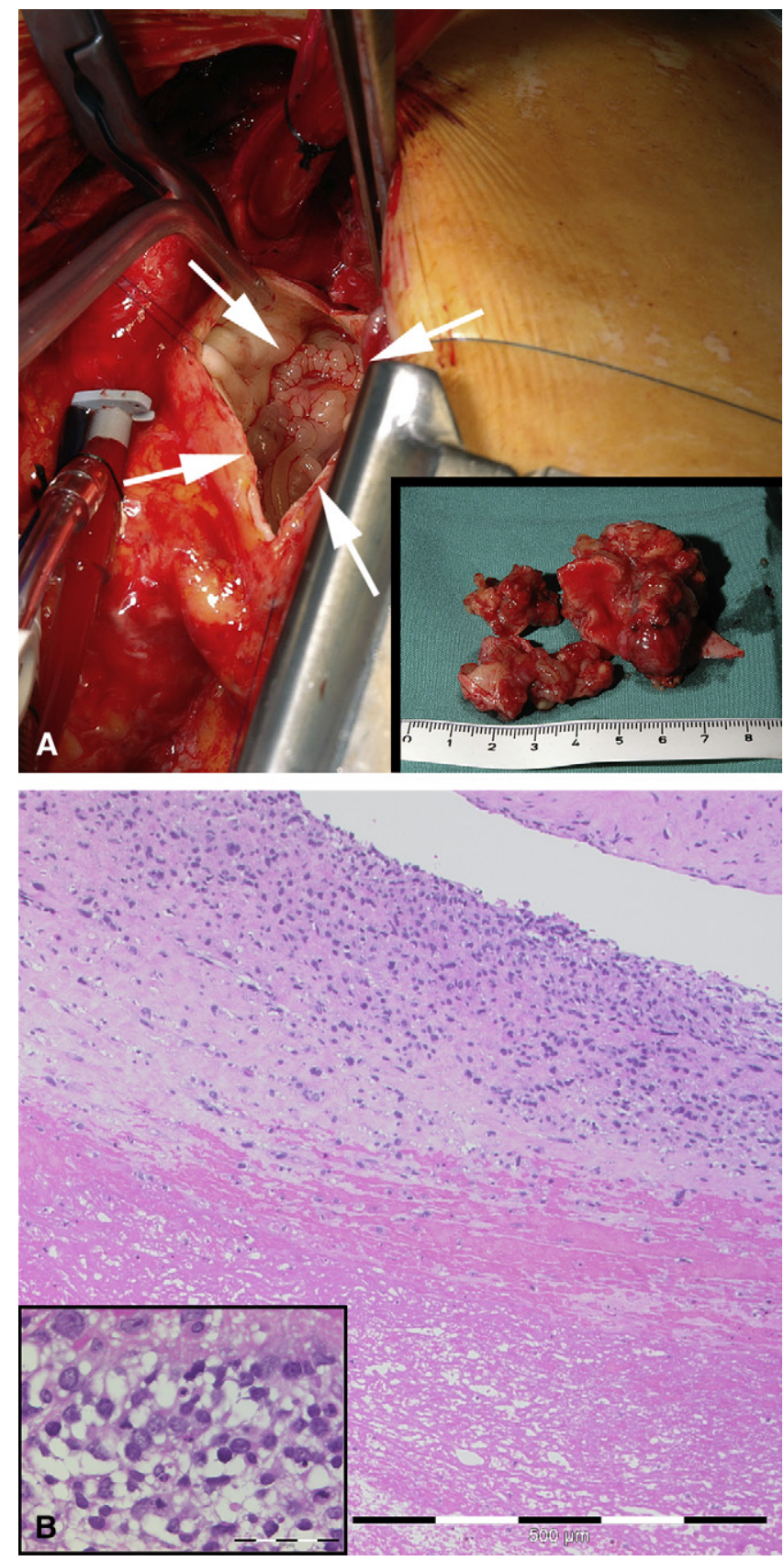

Figure 2. A, Intraoperative situs with the opened wall of the pulmonary trunk uncovers the tumor (arrows) that strongly adheres to the vessel wall. Note the polypoid surface of the lesion that is consistent with the preoperatively detected filiform tails at CT. Note the resected tumor mass of several centimeters in diameter (insert). B, Representative histologic image demonstrating a poorly differentiated mesenchymal tumor with solid (center), epithelioid, and myxoid areas, and tumor necrosis (lower part) and adherent thrombi. The insert shows details of a myxoid area with several highly atypical cells and mitotic figures. (Scale bar $=500 \mu \mathrm{m}$; insert $=\mathbf{5 0} \mu \mathrm{m}$.) 